\title{
MULTIPLE ANTENNA SYSTEMS: FRONTIER OF WIRELESS ACCESS
}

\author{
E. Del Re, L. Pierucci \\ University of Florence, Department of Electronics and Telecommunications, S.Marta 3, 50139 Firenze, Italy, \\ delre@det.unifi.it, pierucci@det.unifi.it
}

\begin{abstract}
Multiple antenna systems are the new frontier for wireless communications including the actually third generation mobile communication systems, called Universal Mobile Telecommunication System (UMTS), the wireless LAN and the wireless PAN up to the future $4 \mathrm{G}$ mobile system focused on seamlessly integration of the existing wireless technologies. The use of multiple antenna systems improves the overall system performance in term of capacity and spectrum efficiency achieving high data rate wireless services. The paper highlights the two main techniques: smart antennas with adaptive beamforming to cancel the interference signals (from other users or multipath) and MIMO systems to exploit the space-time properties of wireless channels.
\end{abstract}

Keywords - Antenna arrays, adaptive beamforming, MIMO systems, space-time processing, wireless communications.

\section{INTRODUCTION}

Recently, in wireless communication systems great interest has been shown for the use of multiple antennas to increase coverage and spectral efficiency.

The popularity of GSM led to a typical site with $120^{\circ}$ sectorized hardware beamforming antenna array increasing the spectrum sharing. The direction and gain of sectorized antennas are fixed and to each beam a distinct channel is assigned.

When the requirements of capacity is exceeded, smart antennas with adaptive digital signal processing are necessary. The smart antenna's beams are not fixed but are dynamic for user and will place nulls in the radiation pattern that cancel interferences. The application of adaptive antenna techniques to fixed-architecture base stations has been shown to offer wide-ranging benefits including interference rejection capabilities. In particular the antenna array should allow the identification of the mobile desired user by using proper DOA (Direction of Arrival) estimation algorithms and improve the signal-to-interference-plusnoise ratio steering the main beam in downlink according the above direction estimate.

In the third generation mobile systems, called Universal Mobile Telecommunication System-UMTS, based on CDMA access, the use of multiple antennas allows improved separation of user signals, decreasing the multiple access interference (MAI) effect. Therefore, space-time processing can significantly increase the capacity of CDMA systems. Diversity combining schemes are proposed such as the Matched Desired Impulse Response (MDIR) approach which performs a spatial cancellation following by a Rake receiver. Joint detection of all bits from all users can overcome the limitations of MAI. In advanced multiuser beamforming approach, smart antennas are combined with interference-canceling multiuser receivers showing a greater performance for the uplink CDMA systems [1].

In UMTS system the use of quasi-orthogonal codes and the multipath of the wireless channel result in a critical MAI [2]. In rich scattering environments the system capacity can be increased by exploiting decorrelation of transmitted signals [1]. In theory, the capacity increases proportionally with the minimum number of antennas at the transmitter or at the receiver. Space-time coding and modulation, and spatial multiplexing MIMO systems exploit different independent parallel transmission channels to provide high data rates increased at no cost in bandwidth or power.

Each multipath reflector is not seen as a drawback to be equalized out, but can seen as an independent path for independent bitstreams .

Lucent decides to beamform into multipath and their Bell Laboratories Layered Space-Time (BLAST) approach [3] [4] shows multipath channel capacity greater than that of a merely noisy channel where, according the Shannon law, the capacity is a function of bandwidth and signal-to-noise ratio. However, the requirement of rich channel scattering is critical and in realistic environment channel coding is needed to approach the MIMO system capacity.

The major source of channel impairment in a spatial multiplexing scheme is co-antenna interference (CAI). To mitigate the degrading effects of $\mathrm{CAI}$, a robust multitransmit multi-receive system using the combination of Turbo decoding principles and V-BLAST, was analyzed in literature [5], [6], called T-BLAST.

The paper first describes smart antenna systems reviewing the concept of adaptive bemforming and DOA estimation to improve the signal-to-interference ratio, highlighting the advantages when they are used in CDMA systems. In the third section an example of DOA estimation particularly optimized for the UMTS environment is considered [7]. Section IV reviews the concepts of MIMO systems: spacetime coded modulation and the iterative space-time layered multiple antenna receiver of the Bell Laboratories. The last 
section shows an example of MIMO system, the combination of the BLAST architecture and the iterative demapping and decoding of the Turbo decoding principle. The performance of this T-BLAST structure was evaluated according to the IEEE $802.11 \mathrm{~b}$ standard requirements for Wireless LAN. Finally, future challenges and issues of MIMO systems are presented.

\section{SMART ANTENNAS}

In the SDMA (Space Division Multiple Access) the capacity of the mobile cellular system increases exploiting the 'spatial diversity' of the users. In particular the radiation pattern of the antenna at the base station should be dynamically modified steering the main beam toward the desired user and by placing the nulls in the pattem corresponding to the interference directions (null steering method). In a personal communication system, this can be achieved using antenna array and an adaptive system is required in order to shape the radiation pattern of the array. The antenna array provides a greater receiving gain than an omidirectional antenna allowing to transmit with a lower power level and to reduce electromagnetic pollution levels. The SDMA techniques increase the overall performance of the radio mobile system in terms of number of users contemporaneously accepted in the same channel. This spatial orthogonality allows to separate the signals from different terminal users even if they are overlapped in the frequency/time domain (FDMA/TDMA techniques) increasing the spectral efficiency. In the case of CDMA access where all the users share the same channel and each user has a different pseudonoise code, the use of smart antennas improves the signal-to-interference ratios allowing to reduce the MAI.

\section{A. Adaptive Beamforming}

In a space diversity system the weigthed sum of the received signals is combined at the output of beamformer to steer a beam toward the user signal and adjust the nulls to reject interference. Different choices of algorithms are possible with a different degree of complexity:

- Based on DOA estimation: if the direction of the desired user is known, the beamformer tries to minimize the output power constrained to maintain a distortionless response in the direction of interest (Minimum Variance Distortionless ResponseMVDR). This method depends strongly on spatial information knowledge (e.g. MUSIC,ESPRIT), is computationally expensive, and the number of users is limited by the number of antenna elements

- Based on training sequence: the beamformer employes a training sequence to minimize the difference between the training sequence and its output in the mean-square sense (MMSE). The use of a training sequence reduce the capacity of the system.

- Blind methods (Constant Modulus-CMA): It works on the premise that the presence of interference and multipath causes distortion on the amplitude of array output which has a constant modulus. It is only effective for constant modulated envelope signals such as GMSK and QPSK.

\section{B. Space-time processing in CDMA}

In the uplink of a wideband direct sequence code-division multiple access (W-CDMA) system the orthogonality of the variable spreading factor (OVSF) channelization codes and scrambling codes (Gold sequence) for each user is affected by severe multipath interferences especially for high-rate users. Therefore, by employing the adaptive antenna array transmitter diversity we can increase the forward link capacity suppressing the MAI. The transmitter antenna weights are modified according the antenna weights generated in the coherent adaptive antenna array diversity receiver.

The downlink weight vector was calculated using uplink measurements, assuming that uplink and downlink frequencies are sufficiently closed to disregard the discrepancies between responses.

A single-user space- time RAKE receiver consists in digital matched filters, a digital beamformer (MMSE type) matched to each path followed by a standard Rake used to phase recombine the delayed signal components. Taking into account the fact that different paths come from different directions it is possible to resolve even path which are irresolvable in the time, due to the low delay spread. The receiver antenna weights are adaptively controlled so that the mean square error between the Rake output signal and the reference signal is minimized using an adaptive algorithm (e.g. LMS,RLS) as shown in fig. 1 [8].

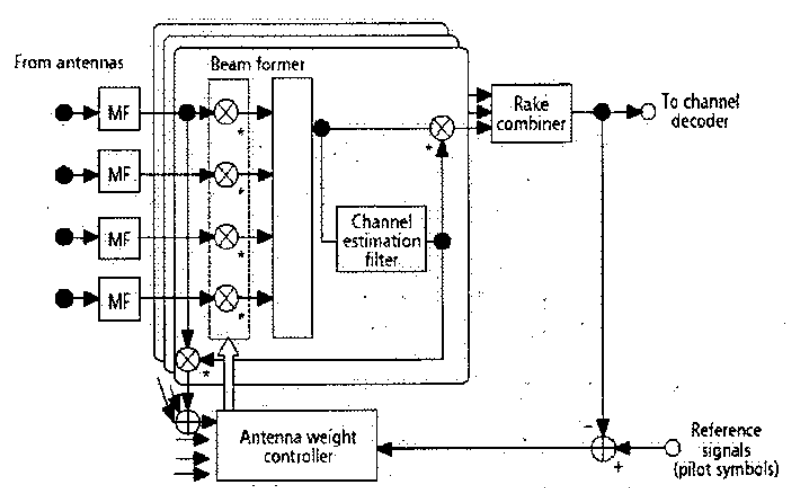

Fig. 1 -Coherent adaptive antenna array MMSE receiver 
In a WCDMA system the conventional matched filter (MF) receiver detects the user's data due to the known spreading code considering the other users signals as noise.

Jointly detection of all data from all users exploits the structure of MAI (weaker and strong users). Among the many multiuser detectors, interference cancellation techniques are relatively computationally efficient.

The Successive Interference Cancellation (SIC) sequentially removes the MAI due to the stronger users before detecting the weaker ones. Parallel Interference Cancellation (PIC) receivers simultaneously cancels all interfering users from the user of interest, before the final symbol decision.

In Selective-Parallel Interference Cancellation the output of a bank of matched filters (one for each signal replica) and Rake is compared with a suitable threshold and assumed reliable or not, depending on its value.

The reliable users are directly detected and cancelled from the whole received signal before making the decision on unreliable signals or replicas. Therefore, the MAI effects of reliable signals (the strongest) on the other signals are cancelled at this second step [9].

In particular multiuser beamforming can decrease the MAI effects combining adaptive antenna array with the interference-cancelling multiuser receiver for the WCDMA uplink [9]. However hardware complexity increases according to the number of accessing users.

\section{DOA ESTIMATION IN UMTS SYSTEMS}

The third generation mobile communication systems, UMTS can support wideband services. UMTS adopts Direct Sequence CDMA access scheme to improve bandwidth efficiency, all users communicate simultaneously in the same band and hence, MAI in addition to multipath significantly degrades performance. In UMTS data rates may vary widely and high rate users may produce severe MAI in the uplink signals.

The use of antenna array in the CDMA system can improve system capacity, quality and reduce severe MAI from high rate users shaping narrow beams to extract the desired signal assuming the DOA's directions are known.

The DOA knowledge is useful to cover in transmission only the interested user, reducing the interference towards the other users and allowing a lower level of electromagnetic pollution, and to determine the position of a mobile user necessary for emergency services.

Conventional DOA methods using the eigenvectors decomposition are based on the assumption that the number of antenna elements is greater than the number of impinging signals (users and replicas). Therefore in multiuser environment with multipath channel these algorithms are not suitable. In CDMA system each user is identified by an unique code orthogonal to the other user codes. Therefore in a optimized approach for UMTS system a DOA estimator (MUSIC or Iterative-MUSIC) is implemented for each user for each replica [7]. The estimators work at the output of matched filters, one for each paths. In this case only one peak of MUSIC spectrum is estimated at a time, providing a high reliability in DOA estimate values and it is more independent from the effect of multiple paths even if the complexity increases.

Unfortunately, the scrambling codes proposed in the 3rd Generation Partnership Project (3GPP) standards have not orthogonality properties to guarantee adequate user identification and the multipath channel contributes to degrade the codes properties. Therefore, the uncancelled MAI decreases the performance of DOA algorithm. However, the simulation results (in Fig.2) show a remarkable behaviour in DOA estimation also in the case of 64 users configuration for a global rate of $3.78 \mathrm{Mb} / \mathrm{s}$ (full chip rate is $3.84 \mathrm{Mchips} / \mathrm{s}$, according the 3 GPP standard).

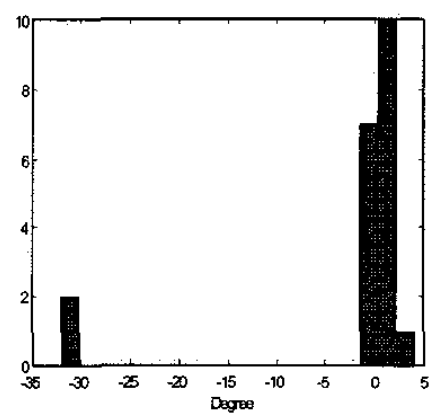

Path $1-\mathrm{DOA}=35.9^{\circ} \mathrm{MSE}=100.93$

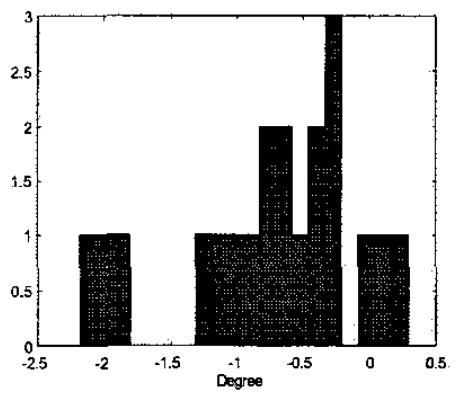

Path $2-\mathrm{DOA}=-30.6^{\circ} \mathrm{MSE}=0.98$

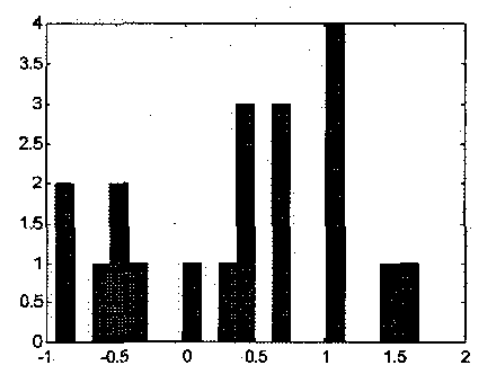

Path $3-\mathrm{DOA}=2.4^{\circ} \mathrm{MSE}=0.68$

Fig.2 - Iterative-Music DOA estimation - Histogram of error estimation around the DOA true value and Mean square error in a and 64 users configuration for three main replicas of desired user in a multipath channel with 6 paths 


\section{MIMO SYSTEMS}

Multiple antennas at the transmitter and receiver provide diversity in a fading environment. By employing multiple antennas, multiple spatial channels are created, and it is unlikely all the channel will fade simultaneously. If statistical decorrelation among antenna elements is provided, this condition can be satisfied by using antennas well separated (by more than $\lambda / 2$ ) or with different polarization, multiple transmit and receive antennas can create independent parallel channels. In rich scattering environments the system capacity can be increased exploiting decorrelation of transmitted signals by using the space-time coded modulation (STCM) and processing of MIMO systems.

Specially in 3GPP downlink the use of multiple transmit antennas using suitable space-time code modulation can achieve the required high data rates.

Generally, in the STCM approach data is encoded by a space-time channel encoder and the output is split into $\mathrm{N}$ streams to be simultaneously transmitted using $\mathrm{N}$ transmit antennas. The received signal at each antenna is a linear superposition of the transmitted signals perturbed by flat fading channel and noise. This coding across spatial channels uses ideas similar to Trellis Coded Modulation (TCM) to define mappings of symbols to antennas. The space-time decoding process at the receiver requires trellis decoding and may have high complexity.

The Bell Laboratories Layered Space-Time (BLAST) approach achieves spectral efficiencies as high as 40 $\mathrm{bits} / \mathrm{sec} / \mathrm{Hz}$ in short-range, rich scattering wireless environment for a fixed total transmit power.

Vertical-BLAST (VBLAST) relies on spatial multiplexing at the transmit multiple antennas and spatial filtering at the receive multiple antennas.

The basic idea is to transmit different signals simultaneously on different antennas and this spatial diversity relies on a rich scattering matrix channel.

V-Blast rather than jointly decoding the signals from all the transmit antenna realizes a beamforming in multipath and first decodes the strongest signal, than cancels the effect of this transmit signal from each of the received signals and then proceeds to decode the strongest second signal of the remaining transmit signals. It is evident the analogy with the multiuser sequentially interference cancellation. The drawback of this method can be envisaged in the order for the sequential estimation and cancellation, from the strongest signal to the weakest one, and in the propagation of errors from one step of detection to the next [4].

\section{T-BLAST ARCHITECTURE}

To mitigate the degrading effects of $\mathrm{CAI}$, a robust multitransmit multi-receive system using the combination of Turbo decoding principles and V-BLAST was analyzed in literature [5], [6], called TBLAST.

In this section, the use of the simplified iterative interference receiver as in [5], and its performance in the specific context of the IEEE $802.11 \mathrm{~b}$ standard is underlined.

The IEEE $802.1 \mathrm{lb}$ standard adopts high data rate with bit rates up to $11 \mathrm{Mbps}$. For achieving data rate greater than 2 Mbps, the IEEE $802.11 \mathrm{~b}$ standard specifies the Complementary Code Keying (CCK) modulation scheme.

The IEEE $802.11 \mathrm{~b}$ complementary spreading codes have code length 8 and a chip rate of $11 \mathrm{Mchip} / \mathrm{s}$. The 8 complex chips comprise a single symbol. By making the symbol rate $1.375 \mathrm{Msps}$, the $11 \mathrm{Mbps}$ waveform ends up occupying the same approximate bandwidth as that for the $2 \mathrm{Mbps} 802.1 \mathrm{lb}$ QPSK waveform. Other IEEE $802.11 \mathrm{~b}$ requirements are:

- It operates in $2.4 \mathrm{GHz}-2.4835 \mathrm{GHz}$ frequency band

- Direct Sequence Spread Spectrum (DSSS)

- Three non-overlapping $22 \mathrm{MHz}$ channels

In the T-BLAST architecture a single data stream is demultiplexed into $M$ substreams, where $M$ is the number of transmitting antennas, and each substream is then encoded into symbols and fed to its respective transmitter. Transmitters 1-M operate in co-channel way at symbol rate with synchronized symbol timing.

It is assumed that the same constellation is used for each substream and that transmissions are organized into bursts of $\mathrm{L}$ symbols. The power radiated by each transmitter is proportional to $1 / \mathrm{M}$, so that the total radiated power is constant and independent of $\mathrm{M}$. A straight forward way to implement coding for V-BLAST is to use the Horizontal Coding architecture shown in Fig.3.

Each layer is encoded separately. In the horizontal encoder operation each layer uses a Parallel Concatenated Convolutionally Code (PCCC) Turbo code, but other error correcting codes can also be adopted.

The optimal receiver processing for a coded BLAST system requires a global ML (Maximum Likelihood) solution, which jointly considers the detection (demodulation), deinterleaving and decoding of error correcting codes. However, due to the high complexity of such an approach many suboptimal techniques have been considered, including linear' processing techniques such as ZeroForcing (ZF) or Minimun Mean Square Error (MMSE) method, and non-linear methods such as Ordered Successive Interference Cancellation (OSIC) [6]..

The performance of these suboptimal detection methods may be improved through iterative detection and decoding, which utilize the decoding results for a second step of processing. 




Fig. 3. Horizontal Coding Turbo-BLAST Scheme.

To extract the desired signal, Zero Forcing (or MMSE)OSIC method at the first step and interference cancellation and Maximal Ratio Combining (MRC) in a layer-by-layer fashion at the subsequent steps are implemented. After the first iteration, where the receiver can start VBLAST detection and decoding from any layer, depending on the observed channel matrix, interference cancellation is performed in a layered-by-layered fashion using the present soft decoding decisions for the already decoded sublayers and the previous iteration decisions for the others.

The parallel Soft-Input-Soft-Output decoders provide the a priori probabilities of the transmitted substreams by using the bit-by-bit MAP decoding method.

The Fig. 4 shows the performance gain of T-BLAST in term of FER versus $E_{b} / N_{0}$ increasing the number of receive antennas in a flat fading channel using 4 transmit antennas, DQPSK modulation and a data rate of $2 \mathrm{Mbps}$ [6].

\section{FUTURE CHALLENGES}

The emerging applications for $3 \mathrm{G} / 4 \mathrm{G}$ wireless systems require of highly variable data rates and guaranteed QoS. On the other hand, WiFi technologies (and in general WLAN and WPAN services) offers higher speed in a small area. A new technology is necessary to provide a seamlessly integration of the existing wireless technologies and cellular systems. To achieve high capacity and spectrum efficiency MIMO systems will play fundamental role. New researches should cover the analysis of behavior of MIMO systems in realistic channel, the study of new space-time coding for multiusers environments, the analysis of new turbo codes techniques to obtain a user terminal at low cost, low power consumption supporting for multiple standards.

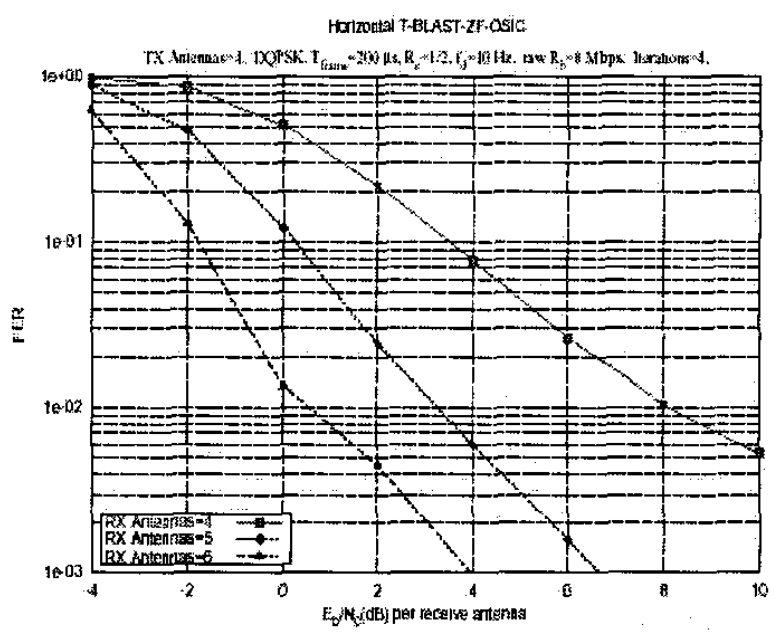

Fig. 4. FER versus $E_{b} / N_{0}$ of Horizontal Turbo-BLAST-ZFOSIC respectively for 4,5,6 Receive Antennas.

\section{REFERENCES}

[1] S. Blostein, H. Leib "Multiple Antenna Systems: their role and impact in future wireless access" IEEE Communications Magazine, July 2003

[2] 3GPP Standard http://www. 3gpp.org release 1999.

[3] G. Golden,C.J. Foschini and R.A. Valenzuela, "Detection algorithm and initial laboratory VBLAST space time communication architecture"', Electronics Letters, January 1999

[4] B.Hassibi, "An Efficient Square-root Algorithm for BLAST", Bell Labs 2000

[5] M. Sellathurai and S. Haykin, "Turbo-Blast for wireless communications: theory and experiments", IEEE Trans. on Signal Processing,V.50,n.10,October 2002.

[6] A. Bernacchioni, E. Del Re, R. Fantacci, L. Pierucci, "T-BLAST Architecture for the IEEE 802.11b context" GLOBECOM 2003, San Francisco

[7] E. Del Re , L. Pierucci, S. Marapodi," On the application of DOA estimation techniques to UMTS system" IEEE 7th Int. Symp. On Spread Spectrum Tech. \& Appli., Praha, Sept. 2-5, 2002

[8] H.Taoka et alii,"Adaptive antenna array transmit diversity in FDD forward link for WCDMA and broadband packet wireless access" IEEE Communications Magazine, April 2002

[9] Del $\operatorname{Re}$ E., Fantacci R., Marabissi D., Morosi S., Armani C.: "Low Complexity Selective Interference Cancellator for a WCDMA Communication System with Antenna Array", IEEE Transactions on Vehicular Technology, Vol-52, n. 4, pp. 1162-1166, Luglio 2003. 